CRfTCA, Revista Hispanoamericana de Filasofla

Vol. XXII, No. 64 (abril 1990): 55-96

\title{
CUESTIONES DE SOBREVIVENCIA
}

ERNESTO SOSA

Brown University

\section{LA PARADOJA}

La vida puede tornarse amarga e, in extremis, puede no valer la pena vivirla. Es más, en ocasiones puede ser mejor dar la vida por una causa más grande. Nada de esto es novedad, por discutible que siga siendo, ya en general, ya caso por caso. Viene a ser novedad que la vida no sea importante en la forma en que habíamos pensado. Nada de resurgimientos del existencialismo ni de noticias de una antigua religión o un nuevo culto: las novedades derivan del más sobrio y tentador de los argumentos filos 6 ficos ${ }^{1}$ y toman, más precisamente, la siguiente forma:

La paradoja: Aunque la vida $V$ sea óptima (en todas dimensiones), y aunque si $V$ se prolongara siguiera siendo óptima, no se sigue que sea mejor prolongarla, ni siquiera para el sujeto cuya vida es $V$.

¿Cuál es el argumento?

La sección II defenderá cierta visión de la naturaleza de las personas y de la identidad personal, y la sección III argumentará luego sobre esa base a favor de la paradoja y reflexionará sobre sus implicaciones filosoficas y sobre las opciones que presenta.

1 El extraordinario argumento de Parfit, 1984, Parte III. 


\section{II. ¿QUÉ CONSTITUYE LA SOBREVIVENCIA?}

Partimos de dos supuestos:

1. Uno no es un alma, la existencia de uno no consiste en la existencia de ningún alma, y la sobrevivencia de uno no consiste en la sobrevivencia de ningún alma.

2. Uno no es ni un cuerpo ni un conjunto de partículas, la existencia de uno no consiste en la existencia de ningún cuerpo o conjunto de partículas, la sobrevivencia de uno no consiste en la sobrevivencia de ningún cuerpo o conjunto de partículas.

La tesis 1 es a menudo defendida mediante argumentos epistemológicos. En otra parte me he opuesto a esto proponiendo otros argumentos. ${ }^{2}$ La tesis 2 se hace plausible por la importancia de la continuidad psicológica para la identidad personal, y por el hecho de que el cuerpo en sí mismo se mantendrá en una relación de superveniencia o de dependencia frente a entidades todavía más fundamentales. ${ }^{3}$

\section{A. Una respuesta aristotélica}

Si no es ni el cuerpo hobbesiano ni el alma cartesiana, ¿qué es entonces la persona y qué debe constituir su existencia y sobrevivencia? Una respuesta dentro de la amplia tradición aristotélica se divide en partes de la siguiente manera: ${ }^{4}$

A1 El perfil personal de una persona en una ocasión es la combinación del perfil psicológico (experiencias, pensamientos, acciones, creencias, deseos, virtudes, habilida-

2 Sosa, 1987.

3 Sosa, 1987 contiene la discusión y defensa de ambas tesis.

4 Para alternativas, compárense, entre otros muchos, los siguientes escritos: Quine, 1953; Perry, 1975; Castañeda, 1975; Chisholm, 1976; Chisholm, 1986; Lewis, 1976; y Wiggins, 1980. Más adelante se citarán otros trabajos. 
des, etc.) y del perfil físico, que incluye todas aquellas propiedades de apariencia o destreza físicas que puedan considerarse significativas para la identidad de una persona.

A2 La existencia de una persona en una ocasión dada consiste en la personificación, por parte un cuerpo, de un perfil personal en esa ocasión.

A3 La vida de una persona a través de una secuencia de ocasiones es una secuencia de perfiles personales personificados, ocasión tras ocasión, interrelacionados por enlaces causales tales como intenciones realizadas, recuerdos verdaderos y los mecanismos físicos de crecimiento y de envejecimiento. La sobrevivencia de una persona a través de esta secuencia consiste en la personificación de un perfil personal por parte de un cuerpo en cada una de esas ocasiones, y en los encadenamientos causales adecuados entre esas personificaciones.

(Un perfil es un conjunto de propiedades y la personificación de ese perfil por parte de un organismo humano equivale a la ejemplificación, por parte de ese organismo, de las propiedades de ese perfil. Para simplificar, hacemos abstracción de la posibilidad de la sobrevivencia a través de la teletransportación, acerca de la cual suspendemos el juicio; lo que significa que suspendemos el juicio acerca de lo tenue que puede ser el componente físico de un perfil personal, en forma compatible, en cualquier ocasión dada, con ese perfil personal que constituye a la persona en esa ocasión. Y esto, además, es compatible con nuestra referencia aristotélica de que ciertas propiedades de un perfil psicológico pueden requerir un cerebro, la sede más intima de ese perfil, para ser personificadas de forma apropiada. Obviamente, el cerebro de un atleta adolescente no conservará todas sus virtudes y habilidades si es transplantado al cuerpo de un octogenario.) 
Recordemos ahora que para el tratamiento de la epilepsia en ocasiones se corta el cuerpo calloso, separando los hemisferios del cerebro. Lo que es sobresaliente es el hecho de que, en un grado significativo, cada hemisferio puede adquirir su propia conciencia independiente.

Consideremos la siguiente serie de posibilidades lógicas e incluso científicas, si bien aún no accesibles tecnologicamente.

P1 Transplantar un cerebro de un cuerpo a otro muy similar (digamos, de gemelos idénticos) de tal manera que el perfil psicológico más directamente personificado por ese cerebro se mantenga a través del transplante, de manera que los recuerdos, deseos, creencias, etc., se mantengan casi inalterados.

P2 Transplantar un hemisferio cerebral de un cuerpo a otro muy similar, destruyendo al mismo tiempo el otro hemisferio (canceroso), de tal manera que, una vez más, el perfil psicológico se mantenga.

Estoy asumiendo que en ambos casos buena parte del perfil personal pueda conservarse en una forma lo suficientemente apropiada como para que la persona pueda sobrevivir a la operación en un cuerpo nuevo. Pero hay una tercerá posibilidad:

P3 Transplantar un hemisferio cerebral de un cuerpo a otrc muy similar, mientras el otro hemisferio (también sano), es transplantado a un tercer cuerpo muy similar (como debe suceder en el caso de los trillizos), de tal manera que en ambos casos se preserve el perfil psicológico.

Consideremos la posibilidad 3. Dada la simetría implícita resultaría arbitrario elegir a cualquiera de los cuerpo-cum-cerebro resultantes, como aquel a través del cual se prolonga la vida anterior. En consecuencia, aparentemente esa vida termina con la operación. Una persona sobrevive a través de una secuencia de estados, cada uno constituido por la personificación de un perfil psicológico, donde la mayoría de ellos se 
entrelaza en una forma causal apropiada; y ahora, además, necesitamos exigir que la secuencia no se ramifique. Esto equivale a decir que, cuando una secuencia se ramifica, una vida termina en el punto de ramificación.

Hay, sin embargo, una alternativa para nuestra explicación de la sobrevivencia como continuidad sin ramificaciones. Se ha argumentado que en lugar de terminar una vida, nuestro doble transplante de hemisferios revelaría vidas paralelas que ya se desplegaban en el cuerpo antes de la operación. Con la operación, estas dos vidas se separarían y divergirían. He aquí la descripción:

LONDRES

$\mathbf{X} \mathbf{X} \mathbf{X}$
TOKIO

Y Y Y

$t_{2}$

\section{$\mathbf{X X X} \quad \mathbf{Y} \mathbf{Y} \mathbf{Y}$}

X X X Y Y Y

X X X Y Y Y

X X X Y Y Y

X X X Y Y Y

XYXYXYXY

XYXYXYXY

XYXYXYXY

XYXYXYXY

XYXYXYXY

$t_{0}$

NUEVA YORK

(Donde P1 corresponde a X X X, y P2 corresponde a Y Y Y. Las 
Xs y las Ys no representan de forma separada; sólo una cadena de ellas representa, y representa la presencia de una parte de cierta vida: una cadena de $\mathrm{Xs}$, la vida de $\mathrm{Pl}$; una cadena de Ys, la vida de P2.)

La operación en el tiempo $t_{1}$ revela que P1 y P2 han estado presentes a lo largo de toda la corriente principal del cuerpo. Esta visión tiene beneficios pero también costos. ${ }^{5}$ Aquí me centraré en un costo. Supongamos que en el tiempo $t_{(0.5)}$ la corriente de conciencia contiene el pensamiento $I_{a}$ : [yo estaré en Londres en $t_{2}$ ]. Así que, en $t_{(0.5)}, \mathrm{P} 1$ y P2 se autoatribuyen la propiedad de estar en Londres en $t_{2}$. Se podría pensar ahora que $P_{a}$ tendrá que resultar tanto verdadero como falso. Pero esto está expuesto a una réplica: "No es $I_{a}$ lo que puede ser verdadero o falso, de la misma manera que ei enunciado 'Estaré en Tokio en $t_{2}$ ' no puede ser en sí ni verdadero ni falso. Lo que es verdadero o falso es, más bien, el pensamiento $I_{a}$ relativo a un contexto tiempo/persona tal como $t_{(0.5)} / \mathrm{Pl} 6 t_{(0.5)} / \mathrm{P2}$ ". Ésta parece ser una réplica prometedora, pero habrá problemas más adelante.

Supongamos que en $t_{(0.5)}$ la corriente de conciencia contiene también el pensamiento $I_{b}$ : [Yo estaré en Tokio en $t_{2}$ ]. En $t_{(0.5)}$ tanto P1 como P2 se han autoatribuido la propiedad de estar en Tokio en $t_{2}$. Pero seguramente en $t_{(0.5)}$ el siguiente pensamiento $I_{c}$ podría ocurrir también: [Nadie está tanto en Londres como en Tokio en $t_{2}$ ]. Así, la simple lógica nos lleva de $I_{a}$ y $I_{c}$ a $I_{b^{\prime}}$ : [Yo no estaré en Tokio en $t_{2}$ ]; y de $I_{b}$ y $I_{c}$ a $I_{a^{\prime}}$ : [Yo no estaré en Londres en $t_{2}$ ]. Pero parece absurdo suponer que alguien pudiera, en cualquier tiempo, estar de acuerdo completamente tanto con $I_{a}$ como con $I_{a^{\prime}}$ o tanto con $I_{b}$ como con $I_{b^{\prime}}$. Sin embargo, tanto $I_{a}$ como $I_{b}$ merecen un lugar en la corriente de conciencia, sobre la siguiente base: si alguien va a estar en cierta ciudad $C$ en un tiempo futuro $t^{\prime}$, posterior a $t$, entonces es lógica y me-

5 El análisis de los costos y los beneficios puede encontrarse en Lewis, 1976, pp. 17-40; Parfit, 1976, pp. 91-96; y en Lewis, 1983, pp. 73-77. 
tafísicamente posible que uno crea correctamente en $t$ que uno estará en $C$ en $t^{\prime}$. (Nótese que uno no necesita tener ninguna justificación para hacer tal predicción: una mera suposición servira.) Por esta y otras razones, la explicación de las vidas sobrepuestas es, a pesar de su brillo lógico, demasiado costosa. La admiro, pero se quedará almacenada mientras proseguimos.

\section{B. Una defensa de la sobrevivencia como continuidad}

De acuerdo con una forma de la explicación de la sobrevivencia como continuidad sin ramificaciones, la explicación llamada del "continuador más cercano" o del "mejor candidato", varios factores se refieren a si una vida continúa o no y en qué sentido. Algunos de estos factores tendrán preponderancia en casos de conflicto. Una vida $V$ continúa en una etapa posterior $S$ si esa etapa $S$ satisface mejor los criterios que otra rival $S^{\prime}$. Es más, un estado $S$ no solamente debe derrotar a todos los estados que compiten por el estatus de continuador de $V$ : debe también llenar ciertos requerimientos mínimos. De este modo debe haber alguna combinación mínima de conexión psicológica y/o de continuidad física entre la etapa $S$ y la vida $V$ para que $S$ esté apropiadamente enlazada con $V$ como una etapa posterior de ella. ${ }^{6}$

Algunas objeciones a esta perspectiva en la literatura incluyen las siguientes.

Objeción 1. Supongamos que una corriente principal de vida $M$ se divide en dos efluentes $E 1$ y $E 2$, donde $E 1$, que es el continuador más cercano, derrota a $E 2$ por el título de extensión de $M$. Supongamos además que si $E 1$ no hubiera existido, entonces $E 2$ habría sido el continuador más cercano de la corriente principal $M$. Entonces $P(E 2) —$ el pro-

6 Wiggins, 1980, pp. 207-209, esboza esta perspectiva y provee referencias adicionales. Wiggins la rechaza, pero Nozick, 1981, capítulo 1, la desarrolla y defiende. Shoemaker da también un uso importante a esta perspectiva en Shoemaker y Swinburne, 1984, pp. 130-132. 
tagonista del efluente $E 2$ - hubiera sido $P(M)$, de acuerdo con la perspectiva del continuador más cercano, sólo si $E 1$ no hubiera existido. Sin embargo, $P(E 2)$ es distinto de $P(M)$, ya que $P(E 2)$ es distinto de $P(E 1)$ mientras $P(E 1)=P(M)$. $\mathrm{Y}$ esto contradice el principio $(\mathrm{N})$ : necesariamente $(\forall x)(\forall y)$ $[(x \text { es distinta de } y) \supset \text { necesariamente }(x \text { es distinta de } y)]^{7}$

Réplica: De hecho, dada esa situación, si $E 1$ no hubiera existido, entonces $P(E 2)$ no habría existido. Es decir, la persona que es de hecho la protagonista del $E 2$ real no habría existido. Ya que esa persona es distinta de $P(M)$, de la persona que es de hecho la protagonista de la $M$ real. Pero si $E l$ no hubiera existido, entonces $E 2$ habría sido el continuador de $M$, y $P(M)$ habría sobrevivido como el protagonista de $E 2$. De acuerdo con esto, $P(M)$, en ese mundo posible, habría sido el protagonista de $E 2$, pero no porque $P(M)$ hubiera sido idéntico a la persona que es en el mundo real la protagonista de $E 2$. Compárese el hecho de que si Nixon (el presidente que más uś grabaciones) no hubiera sido acusado, entonces habría sido presidente en 1975, a pesar de que, en realidad, Ford (el presidente que más gustaba de esquiar) fue presidente en 1975; lo que no implica que si Nixon (el presidente que más uś grabaciones) no hubiera sido acusado, lo habría sido Ford (el presidente que más gustaba de esquiar) en 1975. Entonces, después de todo, no hay contradicción del principio $\mathrm{N}$.

Objeción 2. Bajo la Perspectiva del Continuador Más Cercano hay que trazar una nítida línea (o líneas) que especifique(n) la combinación(es) mínima(s) de conexión psicológica y/o

7 Un argumento muy conocido de Wiggins, 1980. Nótese que la división de una corriente principal en efluentes es ahora compatible con la sobrevivencia, mientras un solo efluente sea dominante. En nuestra concepción usual de la ramificación, en lo anterior y en lo que siga, la ramificación espor definición "equilibrada". De aquí que las "ramificaciones" admitidas por la perspectiva del continuador más cercano se denominen aquí "efluentes" o "continuadores". 
continuidad física requerida para sobrevivir, la mínimamente requerida para que una vida continúe en etapas posteriores. Sin embargo, parece claro que, independientemente de cómo o dónde se trace(n) tal(es) línea(s), las diferencias entre términos colindantes a la(s) línea(s) en cualquier lado habrian tenido que ser triviales. Bajo esta perspectiva, en consecuencia, lo que a uno le preocupa (la propia sobrevivencia) puede depender de algo trivial en sí mismo. ${ }^{8}$

Réplica: Pero, iresulta realmente claro y evidente que hay que trazar una de esas líneas? Que la "vida" de una duna de arena continúe o no, depende de cuántos granos retiene. La duna tiene que satisfacer como mínimo la retención "suficiente" de granos. Pero éste no es un mínimo nítido y no puede volverse nítido salvo por una estipulación arbitraria que defina un nuevo concepto que, aunque estrechamente relacionado con el de la duna, tiene que ser, sin embargo, distinto. Justamente no está claro por qué el concepto de persona tiene que ser tratado de forma diferente. Además, tanto en el caso de la duna como en el de la persona, la existencia misma de la entidad en cuestión debe ser tratada como vaga. Ya que si se toma una entidad dada $E$, ya sea la duna o la persona, y se pregunta si ella (esa misma res) existiría o no en una zona crepuscular de vaguedad, la respuesta no debe ser ni si ni no. Eso debe ser así en cualquier caso en tanto que la instanciación del concepto vago sea un rasgo esencial de $E$, o sea, en tanto que ser una duna sea esencial para la duna y ser una persona sea esencial para una persona. La vaguedad e indeterminación de conceptos esenciales implica la vaguedad e indeterminación de la existencia misma.

No obstante, supongamos que no queremos pagar ese precio y que preferimos abandonar el concepto de persona en favor del concepto de un "exacto". Hay ahora, por estipulación,

8 Parfit, 1976, p. 479. Pero, estrictamente, la opinión en este pasaje no está dirigida directamente a la perspectiva del continuador más cercano, sino a su defensa por parte de Nozick. 
una nítida y minúscula diferencia entre la vida de un exacto y secuencias que se quedan apenas cortas respecto de ella; de aquí que una secuencia que califica y otra que se se queda apenas corta parecieran diferir sólo trivialmente, dependiendo entonces cualquier otra diferencia entre ellas de tal diferencia trivial. Aun admitiendo todo esto, ¿qué se seguiría? ¿Carecería entonces de importancia que sobreviviera el exacto o que, por el contrario, la situación se quedara apenas corta con respecto a ell? Se sugiere una respuesta afirmativa, ya que el que la situación se quede apenas corta o no, habría tenido que depender de una diferencia que es, en sí misma, trivial. Sin embargo, tal razonamiento es falaz ya que lo que de tal modo depende de lo trivial, puede, en sí mismo, no serlo: una advertencia que se fundamentará más adelante.

Objeción 3. Supóngase que la vida $V$ se ha extendido hasta el tiempo $t(n)$ y ha tenido una buena etapa $S$ de continuación de $t(n)$ a $t(n+2)$, pero que en $t(n+2)$ conseguirá un continuador $S^{\prime}$ todavía mejor, a menos que $S^{\prime}$ sea evitada, donde $S^{\prime}$ no es un continuador de $S$. Que el protagonista $P$ de $V$ sobreviva o no en la etapa $S$ en $t(n+1)$ debe por ende aguardar el resultado en $t(n+2)$. Pero esto parece absurdo. Que $P$ sobreviva en $S$ de $t(n)$ a $t(n+1)$ no puede depender de lo que pase después y ser extrínseco a ese intervalo de $t(n)-t(n+1)$. Lo que importa en esa sobrevivencia de $P \operatorname{de} t(n)$ a $t(n+1)$ no puede depender de rasgos extrínsecos a la relación entre la vida $V$ de $P$ de $t(0)$ a $t(n)$ y las etapas constitutivas de $S$ de $t(n)$ a $t(n+1){ }^{9}$

Réplica: Digamos que la vida $V$ de $t(0)$ a $t(n)$ continúa en una etapa posterior $S$ en $t(n+1)$ si $S$ es parte de una secuencia $V^{\prime}$ que va de $t(n)$ a $t(n+1)$ tal que (i) la secuencia $V^{\prime}$ derrota a cualquier rival cotemporal, y (ii) para cualquier $t$ entre $t(n)$ y $t(n+1)$ inclusive, la parte de la secuencia $V^{\prime}$ que va de $t(n)$

9 Compárese Johnston, 1987, p. 68. 
a $t$ también derrota a cualquier rival cotemporal. Esta es una forma de resolver nuestro problema. Sin embargo, no es evidentemente aceptable cuando consideramos la posibilidad de que una secuencia continua $V^{\prime}$ más allá de $V$ puede triunfar sobre todos los competidores por un momento infinitesimal mientras que, después de eso, pierde miserablemente durante cualquier intervalo más largo mientras que otra secuencia continua $V^{\prime \prime}$ es, con mucho, la ganadora a lo largo de todos los intervalos fuera de ese pequeño intervalo inicial. A la luz de lo anterior, parece más razonable admitir que el verdadero continuador de $V$ en $t$ puede ser determinado por lo que ocurra después de $t$.

Tampoco está realmente claro por qué la verdadera sobrevivencia no puede depender de cuestiones en cierto sentido "extrínsecas" a las relaciones pertinentes entre la etapa inicial y la etapa final. El caso de la ramificación antes mencionado es un caso cuestionable. Para hacer una comparación, tómese una bola de nieve constituida en $t$ por cierta cantidad de nieve que, en ese momento, es redonda. Que esa bola de nieve exista en $t$ y sobreviva más allá de $t$ depende decisivamente de que esté lo suficientemente despegada de la nieve que la rodea. Una cantidad redonda de nieve inconsútilmente dentro de un banco de nieve no forma una bola de nieve, y sin embargo, que la nieve redonda esté o no despegada así, es, en un sentido natural, una cuestión "extrínseca". (Me referiré a otros casos similares más adelante.)

Contrarréplica: "La situación en su totalidad es similar al caso de una persona $P 1$ en el mundo real, y una persona $P 2$ en un mundo posible que es similar al mundo real, donde $P 2$ es cualitativamente idéntica a $P 1$ pero difiere con respecto al origen - los padres de $P 2$ no son los padres de $P 1$. Aquí el solo origen establece la diferencia entre $P 1$ y $P 2$. Por tanto, como el origen establece la diferencia entre personas cualitativamente idénticas, entonces 'ser el continuador más cercano' distingue a dos personas cualitativamente idénticas. Lo que no está tan 
claro es si las dos propiedades —el origen y el ser el continuador más cercano_- son análogas.

"Parece una diferencia significativa entre las dos propiedades que el origen de una persona sea una propiedad constitutiva de esa persona, mientras que 'ser el continuador más cercano' no lo es. Entiendo por ello que las cuestiones del origen son cuestiones acerca de la materia de la que está hecha una persona. Un origen diferente implica una materia diferente. Pero que algo sea el continuador más cercano no implica diferencias en su constitución. En consecuencia, si uno pensaba que la cuestión del origen era una diferencia extrínseca entre $P 1$ y $P 2$, entonces la cuestión de 'ser el continuador más cercano' sería significativamente más extrínseca - un tipo o nivel de externalidad diferente. La propiedad extrínseca de 'ser el continuador más cercano' es de un nivel de externalidad tal que no implica diferencia alguna en la constitución del objeto en cuestión. En cambio, la cuestión del límite de una bola de nieve es una cuestión constitucional. Concierne a cuestiones de separabilidad, cuestiones de cómo la bola de nieve que está inconsútilmente enterrada dentro de un banco de nieve podría moverse, etc. Por consiguiente, encontramos natural que tal propiedad extrínseca pudiera determinar si hay ahí una bola de nieve o no, en vista de que es una propiedad constitucional respecto a los límites de la bola de nieve. Pero la propiedad de 'ser el continuador más cercano' es extrínseca de un modo diferente ya que ella no tiene consecuencias similares respecto a la constitución de una persona."

Comentario a la contrarréplica: Estoy de acuerdo en que parece más plausible, al menos inicialmente, dar prioridad a las propiedades "constitutivas" cuando se hace la lista las propiedades esenciales. Pero según la objeción anterior, se supone que las propiedades "constitutivas" involucran la materia de la que está hecha una persona. Es por esto que el origen de una persona es constitutivo, o al menos así se nos presenta: un 
origen diferente implica una materia diferente. Sin embargo, extrapolando a partir de esto, obtenemos un resultado discordante. Puesto que el espacio que rodea a una cantidad redonda de nieve también se supone que es constitutivo de la bola de nieve constituida (en parte) por la redondez de esa nieve. Y aun el que ese espacio esté o no lleno de nieve, no acarrea ninguna diferencia relativa al contenido material de esa cantidad de nieve. Esto no equivale a decir, desde luego, que no hay algún principio de constitución que pondría tanto a los orígenes como al contorno delimitante, por un lado, como constitutivos de varios objetos, planteando a la vez la cuestión de si hay un continuador más cercano o un mejor candidato, por el otro lado, como una cuestión no constitutiva. Pero la apelación al contenido material de la entidad en cuestión no es tal principio y, en verdad, si tal principio existe, creo que aún no ha sido formulado.

Objeción 4. Podemos entender fácilmente la suposición de que una persona $A$ se someta y sobreviva a la implantación, en su cerebro, de la psicología de $B$, mientras que $B$, a su vez, se somete y sobrevive a la implantación, en $s u$ cerebro, de la psicología de $A$. '?Pero cómo es esto posible si el concepto de sobrevivencia personal ha de ser definido o analizado, de manera incompatible, de tal modo que la vida de la persona siga, no a su cerebro, sino a su psicología en cualquier caso de conflicto? ${ }^{10}$

Réplica: En resumen, podemos entender y aun creer que el conocimiento es una creencia verdadera justificada a pesar de que el conocimiento haya de ser definido o analizado, de manera incompatible, de alguna otra forma. Uno esperaría que esto fuera un rasgo inevitable de análisis o equivalencias fi-

10 Compárese Johnston, 1987, pp. 68-69. Sin embargo, ese pasaje no plantea plenamente la objeción que nosotros desarrollamos aquí, ya que su propósito es considerar si en nuestras respuestas a los casos nos guía la teoría del continuador más cercano (el mejor candidato). 
losóficos interesantes. Las elucidaciones filosóficas interesantes generalmente están, a este respecto, muy lejos de estipulaciones de significado triviales o de cosas semejantes. $Y$ aún queda por mostrársenos por qué la sobrevivencia personal debiera ser especial o diferente del conocimiento a este respecto. En ambos casos tenemos un concepto o propiedad $\longrightarrow$ a saber, la sobrevivencia - que se aplica a las personas. Cierto, una es una propiedad que absorbe mucho tiempo en un sentido en que la otra no lo hace. Pero aún no tenemos indicios de por qué esto debe importar. ${ }^{11}$

\section{III. ¿IMPORTA LA SOBREVIVENCIA?}

La sección II ha defendido la perspectiva de que la etapa $S$ en $t$ es una etapa posterior de una vida con una historia temprana $V$ si y sólo si existe alguna mínima combinación de conexión psicológica y/o continuidad física entre $S$ y $V$, y $S$ satisface mejor que cualquier otro competidor en $t$ los criterios relevantes (para el sucesor de $V$ ). Puede ahora argumentarse muy plausiblemente que si nuestra sobrevivencia equivale a lo precedente, entonces no puede tener la importancia que normalmente le damos. Más específicamente, nos vemos llevados a la paradoja.

Si uno se preocupa por la persona cuyo cerebro ha de ser dividido y quiere que esa persona sobreviva a la operación, entonces uno tiene una razón de peso para evitar la ramificación. Y esto parece plausible, incluso en el caso especial en que uno mismo sea la persona en peligro. Por lo tanto, uno tiene una razón de peso para evitar que ambos hemisferios sean transplantados, cada uno en un cuerpo sano por sí mismo. E incluso uno puede tener una razón de peso para asegurar un fin temprano para una $u$ otra de las dos vidas ramificadas - ya que nuevamente uno puede, de este modo, actuar en defensa de alguien cercano, quizá incluso de uno mismo.

11 Una réplica más amplia admitiría la paradoja del análisis. $C f$. Sosa, 1983, pp. 695-710. 
Y aun desde un punto de vista egoísta, si uno está constituido por cierto cuerpo con un cerebro que funciona en cierto tiempo, ¿qué tan razonable sería preocuparse de si la vida de uno continúa a través del transplante de un único hemisferio o de si se ramifica a través de un transplante doble? Parece increíble que pudiera importar demasiado, aun si uno fuera a sobrevivir en un caso pero no en otro. Podría intentarse que pareciera que lo que importa es, cuando más, tener sucesores (causalmente relacionados de manera apropiada con la etapa presente de uno), pero no cuántos. Si el cuerpo de uno está canceroso y lo está también uno de los hemisferios cerebrales, el otro hemisferio debe ser transplantado a un cuerpo sano, con la intención de destruir todos los tejidos cancerosos que se han dejado atrás. ¿Habría alguna buena razón para temer que el hemisferio abandonado resultara sano $y$, por lo tanto, se implantara en un tercer cuerpo sano? Parece que tal temor tiene poco valor.

\section{A. ¿Qué importa en la sobrevivencia?}

Derek Parfit ha argumentado que la sobrevivencia ordinaria (sin ramificaciones) importa poco en sí misma, pero que debemos retener algo importante de dicha sobrevivencia una vez que el requerimiento de no ramificación ha sido descartado. ${ }^{12}$ Lo que podemos retener, lo que sigue siendo intrínsecamente importante para Parfit, es la apropiada relación causal que une entre sí las etapas de una vida mientras ésta no se ramifica. Tómense las ramificaciones efluentes de una vida que se ramifica. La relación causal apropiada une cada una de ellas con la corriente principal de donde se ramifican. Puesto que no nos importará si nuestra vida se ramifica o no, lo que sigue siendo importante en la idea ordinaria de la sobrevivencia personal es justamente esta noción de etapas apropiadas adecuadamente

12 Parfit, 1984, Parte III. 
unidas por la causalidad. Pero considérense las ideas que tenemos ante nosotros: (a) lo que le importará incluso al egoísta será conservar tal influencia causal sobre el futuro; (b) las etapas futuras que le importarán incluso al egoísta radical son simplemente etapas futuras con las relaciones causales pertinentes para su(s) etapa(s) presente(s); y (c) uno también mantiene relaciones causales importantes con otras personas. Estas ideas, (a)-(c), amplían nuestras preocupaciones eminentemente personales. Si no hay nada ontologicamente especial que unifique las etapas de una vida (como lo haría la persistencia de un alma), si lo que unifica nuestra vida son esencialmente ciertas relaciones causales, y si, finalmente, estamos atados a los demás por importantes lazos causales, llegamos de este modo a un acercamiento racional del egoísta con sus projimos. El que nos preocupemos enormemente por la sobrevivencia ordinaria, sea o no en cuanto egoístas, puede acercarnos más a nuestros prójimos, ya que la forma como las etapas de una sola vida están relacionadas es muy similar a la forma como las etapas de la vida del prójimo pueden estar relacionadas. ${ }^{13}$

No obstante, detengamos esta especie de tren de razonamientos en su primera estación, antes de que llegue a las ingeniosas y remotas conclusiones de estaciones posteriores. Se nos ha dicho que lo que importa no es extendernos causalmente sin ramificaciones, sino sólo extendernos causalmente con $o$ sin ramificación. ¿Pero exactamente por qué es la ramificación desdeñable de este modo?

Supóngase que uno está prendado de la forma cúbica y anhela algo con esa forma, aunque le es indiferente el número de sus aristas. Daría lo mismo que fueran ocho o doce aristas, y lo mismo sucedería con dieciséis. La diferencia entre ocho, doce, y dieciséis aristas es para uno una cuestión de poca o ninguna

13 De este modo, Parfit escribe: “podemos comparar el debilitamiento de las conexiones entre la niñez y la vida adulta de una misma persona con la ausencia de conexiones entre diferentes personas". Parfit, 1984, p. 333. 
consecuencia. Lo que es más, nos importa poco el número de lados, y si se nos pregunta qué preferimos, si un objeto hexagonal o uno cuadrado u octagonal, admitiremos sinceramente una indiferencia total. ¿Qué pasa entonces si sucede que ser un cubo consiste en tener seis lados iguales y doce aristas rectas iguales? ¿Puede seguir siendo indiferente para nosotros cuántos lados o aristas tiene nuestro regalo? Ahora se ve que necesariamente el regalo será un cubo si y sólo si tiene seis lados y doce aristas y, verdaderamente, que la cubicidad de nuestro cubo consistirá en ser un sólido regular con seis lados cuadrados iguales y doce aristas rectas. Ya no parece coherente desear un cubo sin que nos importe que tenga seis lados o doce aristas. ¿Cómo se recuperará la coherencia racional? Por alguna de estas dos vías: ya sea valorando menos la cubicidad o valorando más la hexagonalidad y la dodecaedridad. Pero la sola lógica no decidirá nuestra elección.

Compárese ahora la cubicidad con la sobrevivencia (véase la tabla 1).

Tabla 1: Superveniencia, constitución, análisis o similares

En cada caso el hecho o posibilidad en negritas superviene en, o consiste en, o está aproximadamente constituido o analizado por el que está en cursivas.

Mi recibir un cubo

Mi recibir un sólido regular con seis lados cuadrados y doce aristas rectas.

Mi sobrevivir

El extenderse de mi vida sin ramificaciones, por medio de relaciones causales apropiadas que unan etapas presentes y pasadas a etapas futuras.

En cada caso un desideratum en negritas es necesariamente equivalente (por lo menos con cierta plausibilidad y en una primera aproximación) a una posibilidad o hecho en cursivas. $\mathrm{Y}$, en cada caso, antes de captar la equivalencia a uno le importa poco la posibilidad en cursivas. En el primer caso, una 
vez captada la equivalencia, es más natural y apropiado para uno empezar a preocuparse por la posibilidad en cursivas. Sin embargo, se nos advierte contra la posibilidad de responder de este modo en el segundo caso. Se supone que ni debemos reaccionar ni reaccionamos a la equivalencia descubierta al ampliar el alcance de nuestra preocupación para abarcar tanto las cursivas como las negritas. En este caso se supone que la dirección de la influencia normalmente se revierte y en cualquier caso debería revertirse. Así pues, ahora deberíamos dejar de preocuparnos por la posibilidad en negritas, ampliando nuestra indiferencia previa hacia la posibilidad en cursivas, de modo que abarque también la posibilidad equivalente en negritas. Regresaremos a esta cuestión más adelante pero, por ahora, sigamos por mor del argumento. Se ha propuesto que ampliemos la tabla como sigue (véase la tabla 2).

Tabla 2: Superveniencia, constitución, análisis o similares

En cada caso el hecho o posibilidad en negritas superviene en, o consiste en, o está aproximadamente constituido o analizado por el que está en cursivas.

Mi recibir un cubo

Mi recibir un sólido regular con seis lados cuadrados y doce aristas rectas.

Mi sobrevivir

El extenderse de mi vida sin ramificaciones, por medio de relaciones causales apropiadas que unan etapas presentes y pasadas a etopas futuras.

Mi "sobrevivir"

Que mi influencia se extienda por medio de relaciones causales apropiadas que unan etapas presentes y pasadas de mi vida a etapas psicológicas futuras, con o sin ramificación.

Aunque la auténtica sobrevivencia puede describirse mejor que en esta tabla, al menos en una primera aproximación, no es, sin embargo, la auténtica sobrevivencia lo que realmente nos 
importa o debiera importarnos: bien pensado, realmente nos importa y debiera importarnos sólo la "sobrevivencia" como se ha definido.

Sin embargo, ¿por qué habría esto de merecer nuestro interés? ¿Tiene semejante "sobrevivencia" algún mérito independientemente de estar o no contenida en la sobrevivencia ordinaria? ¿Acaso nuestra influencia causal sobre el futuro es siempre algo que tiene una importancia propia e intrínseca para nosotros? La propuesta de la "sobrevivencia" depende del valor intrínseco que se encuentre en una noción como la de extender la influencia causal de uno sobre el futuro. Pues supongamos que esta "sobrevivencia" no es propiamente un desideratum en sí misma, que no tiene ningún valor intrínseca y separadamente de su inclusión en la auténtica sobrevivencia. Luego, por paridad de razonamiento, necesitaríamos concluir que la "sobrevivencia" tendrá el mismo destino que la no-ramificación.

Permítasenos considerar, en consecuencia, si es realmente la "sobrevivencia" lo que debería importar o si debemos preocuparnos por que nuestras etapas psicológicas presentes influyan apropiadamente sobre el futuro, sin que lo apropiado de esta influencia dependa en lo absoluto de que se eluda la ramificación. ¿Puede haber intrínsecamente méritos obvios en tal influencia causal? Supóngase una máquina "replicante" que produce tantas réplicas como puedan desearse con la relación causal correcta con nuestra psicología. ¿Habría una gran demanda por esa máquina? Supongamos que pudieran resolverse todos los problemas prácticos: e.g., que todas las réplicas pudiesen ser enviadas inmediatamente a galaxias distantes. Aun así, ¿es esa mera multiplicación de la influencia causal de uno un desideratum obvio?

¿Acaso hemos malinterpretado la propuesta de que la "sobrevivencia" reemplace a la sobrevivencia como el objeto apropiado de nuestra preocupación? Quizá la proposición no es que cualquier instancia de la extensión de la influencia causal de 
uno deba tener su propia medida de valor, valor que deberá entonces acumularse a través de múltiples réplicas. Quizá la propuesta es más bien ésta: que en el centro de nuestro interés egoísta yace más bien el valor de ampliar la influencia causal de nuestra psicología sobre el futuro por lo menos una vez. Eso es lo que realmente buscamos o deberíamos buscar por su propio valor intrínseco. Y esta meta es compatible con la ramificación, mientras que no se sigue que más y más ramificaciones nos darán más y más de lo que queremos. Esta meta, en consecuencia, parece no comprometernos a repetir los usos del replicante, ni aun prima facie y revocablemente. Ya que de la conveniencia de tener al menos un efluente no se sigue nada acerca de la conveniencia de múltiples efluentes.

La respuesta anterior me parece enteramente coherente dentro de una lógica estricta. Sin embargo, lo que es lógicamente coherente no es necesariamente razonable o siquiera sano, y queda por verse si podemos aceptar esa respuesta. Supongamos que (a) es conveniente tener por lo menos una cosa de cierta clase de cosas o al menos una unidad de cierta clase de objetos sin que (b) la clase de cosas o la clase de objetos tenga alguna medida de valor para uno sólo por ser esa clase de cosas o esa clase de objetos. De ser así, entonces (a) no implicaría que cada vez más de esa clase de cosa o esa clase de objeto será cada vez mejor. Uno puede, claro, tener "demasiado de una cosa buena", por una diversidad de razones. En general, la sobrepoblación y el sobreabastecimiento pueden tener efectos o pueden formar patrones más amplios que anulen al bien contenido en la clase de cosa u objeto involucrado. Así pues, podría ser razonable desear que haya al menos una cosa sin comprometerse con el dictum "mientras más, mejor". La anulación por "sobreabastecimiento" del bien contenido en una clase de cosa u objeto, en una mercancía, parece no estar disponible, sin embargo, donde la mercancía es la influencia causal de la corriente de vida de uno sobre el futuro. Al menos, parecería posible construir ejemplos en los que aparentemente 
no hubiera ningún factor de anulación que se accionara, con crecientes líneas de tal influencia causal, con cada vez más efluentes brotando de la corriente principal de la vida de uno; ningún factor semejante cuya presencia podría entonces anular al bien contenido en tal extensión causal per se.

El razonamiento precedente asume que apelar a la presencia o ausencia de la auténtica sobrevivencia no está permitido. Por supuesto, con más de un efluente que brote de la corriente principal de nuestra vida, perderíamos nuestra propia auténtica sobrevivencia, y eso, creo, es un importante factor de anulación. Sin embargo, estamos considerando ahora la simple extensión de la influencia causal de nuestra psicología sobre el futuro, con o sin ramificación, como lo que verdaderamente importa en el centro de nuestros intereses egoístas. Y esto ha sido propuesto, precisamente, por quienes desean minimizar la importancia de la auténtica sobrevivencia. De modo que ahora parecería incoherente para estas personas apelar a la importancia de la auténtica sobrevivencia como un factor que puede anular al bien contenido en la extensión causal de nuestra psicología per se, explicando así cómo podemos desear razonablemente que nuestra psicología se extienda al menos una vez sin desear cada vez más extensiones de ella conforme surjan las oportunidades.

Tomemos otro ejemplo. En una sociedad polígama alguien puede querer tener al menos una esposa mientras que razonablemente puede detenerse ante el deseo de tener cada vez más esposas. Y esto es fácil de entender ya que la cercanía puede verse amenazada por la multiplicidad. Así podemos entender cómo puede haber valores que anulen para nosotros el valor del matrimonio, llevándonos a desear al menos una esposa, pero no más de una o, quizá, cuando más, un número limitado.

¿Qué hay de la propuesta de que lo que verdaderamente importa en el centro de nuestros intereses egoístas es la "sobrevivencia", o sea, extender causalmente nuestra psicología hacia el futuro; de que lo que verdaderamente nos importa, o debería 
razonablemente importarnos, desde la perspectiva de nuestra corriente principal de vida, es simplemente que esta vida tenga por lo menos un efluente en ocasiones futuras? Comparemos esto con el hecho de que a uno le debe importar tener por lo menos una esposa, pero no más de una. Comparando las dos, aparece una diferencia importante: podemos ver las clases de valores que se verían amenazados por tener demasiadas esposas; pero yo no tengo ningán indicio de qué valores importantes estarian en peligro por la existencia de más de un efluente, en casos como el de nuestra máquina "replicante", para referirnos solamente a un ejemplo; no es fácil ver qué valores estarían en peligro en tales casos, excepto solamente para la auténtica sobrevivencia del protagonista de la corriente principal. Y esto obviamente resulta inútil para la defensa de la "sobrevivencia" por encima de la sobrevivencia.

Por lo común, la motivación que surge del interés propio no contiene germen alguno de explicación del supuesto valor intrínseco de tal influencia causal. De acuerdo con esto, parece dudoso que el hecho de influir sobre el futuro, en sí mismo, sea para nosotros un desideratum importante. Supongamos, por el contrario, que cualquier valor de los que surjen del interés propio que pueda hallarse en tal influencia causal deriva del hecho de que está contenido en nuestra sobrevivencia personal ordinaria, de tal manera que nuestra auténtica sobrevivencia necesariamente lo requiere. Si así es como defendemos el valor de la influencia causal, entonces la misma estrategia servirá para defender la no-ramificación.

$\mathrm{Si}$, por otra parte, descartamos la influencia causal como una relación que agrupa a una secuencia de estados en una sola vida deseable, ¿qué puede entonces unificar una secuencia de estados como las etapas de una sola vida deseable? Antes degradamos a la sobrevivencia cabal, sin ramificaciones, en favor de una débil relación de influencia causal apropiada que admite la ramificación. La estrategia ahí era minimizar la importancia de la auténtica sobrevivencia, y remplazarla, dentro 
del interés por nosotros mismos, por un componente parcial de la sobrevivencia ordinaria: a saber, por entrelazamientos causales de una clase apropiada entre etapas psicologicas, aun cuando esto no unifica necesariamente las etapas entrelazadas en etapas de una vida individual. Como sabemos, no logra hacer eso, por ejemplo, en cualquier caso de ramificación, esto es, en cualquier caso de ramificación auténtica y equilibrada, razón por la cual no puede ya servir como un objeto de auténtico interés propio. Sin embargo, fue propuesto como el más valioso sustituto.

Ahora la estrategia es enfatizar una vez más que, de acuerdo con el razonamiento que estamos examinando, debemos minimizar la importancia de la unidad de una vida como la vida de una persona individual que perdura. Ser la misma persona en el futuro carece de importancia y aun la influencia causal parece disminuida. ¿Qué es lo que debe conservar alguna importancia? Tal vez sólo alguna parte del concepto de influencia causal apropiada: la "semejanza" junto con la continuidad espacio-temporal, aun cuando esta relación no logra unificar los estados psicológicos como etapas de una vida individual (fracaso que se encuentra ya en la relación de influencia causal apropiada).

Haciendo a un lado ambos requerimientos, el de la no-ramificación y el de la influencia causal, añadimos un cuarto par a nuestra tabla:

Tabla 3: Superveniencia, constitución, análisis o similares

En cada caso el hecho o posibilidad en negritas superviene en, consiste en, o está aproximadamente constituido o analizado por el que está en cursivas.

Mi recibir un cubo

Mi recibir un sólido regular con seis lados cuadrados y doce aristas rectas. 
Mi sobrevivir

El extenderse de mi vida sin ramificaciones, por medio de relaciones causales apropiadas que unan etapas presentes y pasadas a etapas futuras.

Mi "sobrevivir"

Que mi influencia se extienda por medio de relaciones causales apropiadas que unan etapas presentes y pasadas de mi vida a etapas psicológicas futuras, con o sin ramificación.

\section{Mi "cuasisobrevivir"}

Que la secuencia de estados psicológicos constitutiva de mi vida pasada y presente se vea seguida por estados psicológicos posteriores con cierta "semejanza", sea que estén o no acompañados ya sea por una influencia causal o por una no-ramificación.

La "cuasisobrevivencia" puede ser propuesta como lo que verdaderamente importa, a la luz de nuestras dudas sobre la influencia causal.

Sin embargo, ¿́por qué habría de ser deseada por sí misma la "cuasisobrevivencia"? ¿Porque es lo que permanece de la sobrevivencia una vez que eliminamos los requerimientos de la influencia causal y de la no-ramificación? Como ya hemos visto, esta respuesta no apoyará ninguna propuesta semejante acerca de lo que verdaderamente importa en las motivaciones que surgen del interés propio. De nada servirá que sea esto lo que quede de nuestra noción de sobrevivencia auténtica — para la sobrevivencia ondinaria casi cualquier precio es demasiado alto. Ya que nunca sería suficiente para salvar a alguna propuesta del destino que supuestamente sufre el concepto de noramificación y el concepto mismo de sobrevivencia auténtica y ordinaria -el destino de ser degradado en nuestro interes cuando se encuentra que contiene un componente lógico con un mérito intrínseco indiscernible. Por lo tanto, para que la “cuasisobrevivencia" importe en el presente contexto, debe importar por su propio valor intrínseco y no por haber estado 
contenida en la preciada sobrevivencia ordinaria. Así, debemos considerar el valor de la "cuasisobrevivencia" en sus propios términos. ¿Es realmente la "cuasisobrevivencia" lo que importa por su propio valor intrínseco? Permítasenos imaginar una máquina que produce en nuestra más cercana proximidad espacio-temporal otros seres humanos con propiedades relacionadas al azar con las nuestras. ¿Querría uno un doble de uno mismo como la siguiente creación al azar? Confieso no tener deseos de algo semejante ni, pienso, los tendría la mayor parte de la gente. ¿Estamos simplemente perdiendo algo importante? Si es así, necesitamos mostrarlo. La relación de "semejanza" junto con la continuidad espacio-temporal parece no ser importante, por lo menos intrínsecamente o por sí misma. Si nuestra vida es infeliz, entonces la semejanza de etapas futuras no puede ser un objeto de deseo apropiado — tal semejanza no puede, por sí misma, servir como una fuente de valor para nuestra vida. Si en nuestra vida hemos experimentado intenso sufrimiento y poco de lo demás, desearemos para etapas futuras diferencia y no semejanza. Lo que uno quiere para esas etapas futuras, en relación con las etapas presentes, parecería ser, principalmente, una alta calidad de vida y no necesariamente (a) una derivación causal o (b) semejanza.

Mediante esta serie de razonamientos hemos llegado finalmente a una estación en la que sólo la utilidad contenida en las etapas o estados psicológicos importa de modo fundamental: cualquier etapa de cualquier vida, o al menos cualquier etapa futura de cualquier vida con dicha etapa. Lo que es más, de este modo podemos llegar a una estación más radical todavía, donde ni siquiera pueda importar si esos estados futuros constituyen etapas de alguna vida; sólo importa que sean estados con un contenido de alta calidad.

Finalmente, no hemos encontrado ninguna serie de razonamientos para volver a desiderata más plausibles, excepto yendo de regreso a la estación original de partida, donde lo que importa es nuestra cabal sobrevivencia personal. $\mathrm{Y}$ si esto re- 
quiere de la no-ramificación, tal vez no haya, después de todo, ningún camino plausible para evitar estos requerimientos. Hasta donde hemos sido capaces de determinar, ningún punto intermedio ofrece un refugio coherente y estable.

Queda aún, sin embargo, una pregunta recurrente: ¿Qué puede decirse a favor de la tesis de que lo que fundamentalmente importa no es la auténtica sobrevivencia, sino algunos sustitutos más débiles? ¿Por qué esta tesis ha probado ser tan influyente? ¿Qué razones se han ofrecido realmente contra la importancia de la sobrevivencia?

\section{B. Principales argumentos en contra de la sobrevivencia}

Dos interesantes líneas de argumentación han llevado a la conclusión de que la sobrevivencia es una mercancía devaluada. En la primera línea de argumentación la importancia de la sobrevivencia personal puede ser solamente "derivativa", y debe derivar del interés que conferimos a nuestros proyectos "noprivados". ${ }^{14}$ En la segunda línea de argumentación lo que importa en la sobrevivencia personal de una vida continua (a) no puede depender de rasgos "extrínsecos" a la relación pertinente entre sus etapas, y (b) no puede depender de ningún hecho "trivial". ${ }^{15}$ Echemos un vistazo más detallado a ambas líneas de argumentación.

14 Perry, 1976, pp. 67-91.

15 Parfit, 1984, Parte III, passim, e.g., p. 267 y s8.; que reconoce su deuda con los escritos de Bernard Williams; véase, e.g., Williams, 1973, p. 20. Parfit sugiere que los requerimientos (a) y (b) son plausibles, aunque estrictamente su argumento es ofrecido a aquellos que aceptan la perspectiva de Williams, y el mismo Parfit no apoya (a) y (b) explícita y categoricamente. Por supuesto, si no es asi como se muestra que la sobrevivencia está devaluada, ientonces cómo? Esta pregunta tiene carácter de urgente a partir de la sección $A$ anterior, y cualquiera que tampoco apoye el argumento de la sección $\mathrm{B}$ nos debe algún argumento altemativo. 


\section{Primera línea de argumentacion:}

Premisa 1. El valor de la sobrevivencia personal debe ser "derivativo", y debe derivar del interés que conferimos a nuestros proyectos "no-privados".

Premisa 2. Pero nuestros proyectos "no-privados" serían algunas veces fomentados aun mejor por un equipo de "sobrevivientes" duplicados que por un sobreviviente solitario.

Conclusión. Algunas veces sería mejor para nuestra vida que se ramificara (produciendo dos o más "sobrevivientes" duplicados), a que no se ramificara (produciendo sólo un sobreviviente solitario).

Que la sobrevivencia no importe de manera fundamental se sostiene asumiendo que los desiderata que fundamentalmente importan deben ser "no-privados" —en el sentido en el que "que un artículo se escriba" $(N)$ es ahora un proyecto mío noprivado, mientras que "que yo escriba un artículo" $(P)$ es más bien un proyecto privado. La importancia para mí del proyecto $P$ y de cosas semejantes es derivativa de una importancia más fundamental para mí de proyectos como $N$. La importancia para uno mismo de la propia sobrevivencia derivaría normalmente del hecho de que la sobrevivencia propia está normalmente en la mejor posición para fomentar los proyectos no-privados presentes de uno. Pero debe haber poco o nada de donde escoger, entre, por un lado, la auténtica sobrevivencia personal (que requiere, según las concepciones más plausibles, la noramificación) y, por el otro, el "sobrevivir" a través de dos o más ramificaciones efluentes equilibradas. Pues en ambos casos los proyectos no-privados presentes de uno podrían ser fomentados al menos igualmente bien tanto por varios sucesores "sobrevivientes" como por uno solo.

¿Por qué se pensaría que los proyectos privados deben tener su real y apropiada importancia derivativamente a partir de la de los proyectos no-privados? Esto puede inferirse a partir de 
una restricción relativa al valor fundamental (RVF) como la que sigue.

RVF Los principios fundamentales de valor no deben hacer referencias esenciales a ningún individuo particular o a eventos reales: deben ser puramente generales.

De este modo, el Principio de Utilidad calificaría, mientras que ningún Principio de Egoísmo tal como "Yo importo más" podría considerarse como fundamental.

RVF apoyaría el razonamiento según el cual la sobrevivencia propia no puede ser postulada de forma racional como fundamentalmente valiosa. La importancia de la sobrevivencia propia debe derivar, en cambio, de sus contribuciones a otros desiderata, que son, por ende, más fundamentales. Y de esto se sigue que, como es de imaginar, debe haber poco de donde escoger entre nuestra auténtica sobrevivencia y nuestra "sobrevivencia" a través de dos o más ramificaciones efluentes. En verdad, esto último debe ser, como podemos imaginar, un resultado más deseable. Ello depende del carácter de los proyectos no-privados y racionales de uno. Supóngase que incluyen el desideratum $(D)$ según el cual las preferencias de la gente (actitudes a favor, valoraciones positivas) se satisfacen en la medida en que pueden ser comprendidas. El deseo de comida cuando se tiene hambre, el deseo de bebida cuando se tiene sed, el deseo de huir cuando se tiene miedo - tales deseos tienen ahora una exigencia derivativa de satisfacción racional. Por revocable que pueda ser, esta exigencia es, no obstante, real y apremiante, y es racional que lo sea. Algunos de estos deseos bien pueden ser reducidos o eliminados en favor de una vida más coherente. Ciertos temores pueden ser tontos, por ejemplo, y causar mucho sufrimiento innecesario. Uno saldría mejor librado sin ellos y trataría de eliminarlos. Pero otros deseos son firmemente inherentes a la fisiología o a la psicología humanas, y el absurdo residiría, no en su presencia, sino en el intento de eliminarlos. Así puede resultar absurdo argumentar 
en contra del hambre o la sed, ya que son fuertes y tienen bases fisiologicas.

Desde luego, alguien podría tomar en cuenta más a la "sobrevivencia" que a la sobrevivencia, y esto podría ser como el hambre o la sed en su primitividad y en su resistencia a la persuasión racional. Sin embargo, me parece que lo que a la mayor parte de nosotros los humanos de hecho nos preocupa en ese sentido primitivo es la sobrevivencia más que la "sobrevivencia". Y yo no veo que se haya mostrado que esto es irracional, especialmente para aquellos que ni siquiera han pensado en los recientes razonamientos filosóficos sobre este tema. Tómese ahora a aquellos que han seguido tales razonamientos y que han salido con la intuición de que en los casos de ciencia ficción se preocuparían más por la "sobrevivencia" que por la sobrevivencia. Supóngase que van más allá y se encuentran ahora mismo preocupándose más por la "sobrevivencia" que por la sobrevivencia. No tengo absolutamente ninguna objeción contra esta reacción. Simplemente no es la mía. Lo que todavía encuentro oscuro, sin embargo, es la afirmación de que incluso para tales filosofos es la "sobrevivencia" lo que realmente importa (o debería importar) en la sobrevivencia. Compárese la siguiente forma de describir el problema, la cual empieza comparando a la "sobrevivencia" con la descendencia. Tener descendencia es, en ciertos sentidos, más similar a tener "sobrevivientes" de lo que lo es esto último con respecto a sobrevivir. ¿Por qué no decir que para los filósofos en cuestión se vuelve preferible tener descendencia o sucesores de cierta clase que sobrevivir? Supongo que uno podría incluso comparar esto con casos en los que se tenga que elegir entre sobrevivir y tener dos hijos que serán muy semejantes a uno, donde uno mismo deja de existir o los dos hijos dejan de existir (o ni siquiera llegan a existir —es posible comparar aquí casos diferentes). Me imagino que aquellos que no se preocupan tanto por sobrevivir como por "sobrevivir" optarían también por los hijos, al menos en algunos de los casos que puede uno construir aquí, 
incluso a costa de su propia sobrevivencia. Pero yo describiría esto como la manifestación de que están más orientados hacia la descendencia que hacia la sobrevivencia, y no diciendo que lo que realmente les preocupa en su propia sobrevivencia es justamente su "sobrevivencia" —al menos no si al decir esto se está diciendo que a uno le preocupa la sobrevivencia sólo derivativamente, sólo porque contiene la verdaderamente apreciada "sobrevivencia".

No es que yo rechace la idea de que la "sobrevivencia" está, en algún sentido, en la sobrevivencia (como de algún modo lo está parte de su contenido lógico). $\mathrm{Ni}$ tampoco, por supuesto, niego que a dichos filosofos les importe la "sobrevivencia". Y así, en ese sentido, al preocuparse por la "sobrevivencia" se preocupan por algo en la sobrevivencia. Pero aun así no está claro que la "sobrevivencia" sea todo lo que a ellos les importa en la sobrevivencia. ¿No se preocupan también por la sobrevivencia misma? Si es así, no pienso que dichos filósofos remplacen o tengan que remplazar la sobrevivencia por la "sobrevivencia" y que les preocupa la sobrevivencia sólo porque contiene la preciada "sobrevivencia". Después de todo, ¿por qué no podrían continuar preocupándose, primitiva y no-derivativarrente, por la sobrevivencia misma en la mayor parte de las circunstancias, aunque prefirieran la "sobrevivencia" a la sobrevivencia en ciertos casos de ciencia ficción en los cuales se debe hacer una elección? El que la sobrevivencia continúe importándoles, primitivamente, no está más amenazado por tal respuesta a los casos de ciencia ficción, de lo que lo estaría por la respuesta de aquellos que optaran por los hijos más que por su propia sobrevivencia en los casos en los que era obligado hacer una elección. Y esto me parece así, incluso si existe una relación entre su propia vida pre-reproducida y las vidas de los hijos, relación que es análoga, si no idéntica, a la relación entre su propia vida pre-reproducida y su vida post-reproducida, e incluso si encontramos que esta relación es la que está implícita en el interés por nuestra descendencia. 
¿Podría nuestro instinto de autoconservación constituir un deseo relativamente resistente a la persuasión o a la argumentación, parecido al hambre y a la sed? De ser así, podría estar más racionalmente fomentado que socavado. Y esto produce una respuesta suficiente a la primera línea del argumento en contra de la importancia de la sobrevivencia personal. Sí, la importancia de la sobrevivencia personal puede ser en un sentido derivativa (puede ser), pero entonces se deriva de su propia existencia obstinada y no sólo de que es un medio para la satisfacción de algún otro deseo "antecedente" — en el sentido en que la importancia de un boleto de avión puede derivarse de que nos permite volar a cualquier parte, lo que puede ser en sí mismo un desideratum si nosotros mismos vamos a ser el boleto. Mientras el deseo de sobrevivir se mantenga obstinadamente, se mantendrá obstinadamente su importancia, y lo hará racionalmente, por lo general de cualquier modo: al menos tan racionalmente como lo hace, comúnmente, el deseo de apagar la sed. Aun admitida, esta defensa no es ninguna prueba, ya que en su estado actual serviría también para defender obstinados deseos patológicos. Pero aún se nos debe mostrar que el deseo de sobrevivencia se parece más a un deseo patológico que a un sano deseo de comida o bebida. Cierto, si la sobrevivencia requiere de la no-ramificación exigirá un tipo de control de población, pero ni siquiera todos los utilitaristas radicales favorecerían el crecimiento ilimitado de la población.

En cualquier caso, es importante extraer de forma más completa las consecuencias de la RVF, que incluyen no sólo el rechazo del egoísmo como valor o norma fundamental, sino también, sobre las mismas bases, un rechazo paralelo de la lealsad. Quizá la RVF permite reinstalar a la lealtad como una virtud con algún tipo de justificación derivativa, quizá mediante su valor utilitario en cualquier grupo bien ordenadu. Pero queda aún por ver qué tan buena o mala sería la "leaìtad" para uno mismo, qué lugar habría ahí para la autocnnservación v nara el deseo de auténtica sobrevivencin úentro de tol vistema. 
2. Segunda línea de argumentación:

Premisa 1. Lo que importa en la sobrevivencia personal de una vida que continúa (a) no puede depender de rasgos "extrínsecos" a la relación pertinente entre sus etapas, y (b) no puede depender de ningún hecho "trivial".

Premisa 2. (Supóngase que la corriente principal $M$ de una vida llega a cierta coyuntura $J$. Y considérese de qué manera esa corriente principal puede estar relacionada con un efluente $E$ que fluye a partir de la corriente principal $M$ en la coyuntura $J$. ¿Hay otros efluentes "equilibrados" $\operatorname{con} E$, que fluyen también a partir de $M$ en $J$ ?) Que haya o no tales efluentes es un problema que debe ser "extrínseco" a la relación entre $M$ y $E$, en virtud de que $E$ debe constituir la continuación de $M$; además, es un problema que debe ser "trivial" para el protagonista de esa corriente principal $M$.

Conclusión. Lo que importa en la sobrevivencia de la vida $M$ a través del efluente $E$ más allá de la coyuntura $J$ (o, lo que es equivalente, lo que importa en la sobrevivencia del protagonista de $M$ a través de $E$ ) no puede conllevar o depender de si hay o no otros efluentes que brotan de $M$ en $J$ y equilibrados con $E$.

Las explicaciones de la sobrevivencia personal tienden a requerir continuidad bien física, bien psicologica, o bien ambas. Podría decirse que para que la vida de una persona se prolongue mucha de la psicología de esa persona debe ser preservada causalmente; o buena parte de su asiento físico debe ser preservado; o ambas cosas. Sin embargo, como hemos visto, "mucha" de tal continuidad psicologica o física puede relacionar a una corriente principal de vida con más de una ramificación efluente. Dado que las diversas ramificaciones no pueden ser una y la misma, y dado que ninguna diferencia significativa puede justificar que se privilegie a alguna de ellas como la corriente principal continua, se dice que la vida de la corriente 
principal termina en el punto de ramificación donde dos nuevas líneas, los efluentes, comienzan.

Tómese de nuevo un caso de ramificación, en el que dos ramificaciones efluentes $B 1$ y $B 2$ se originan en una corriente principal $M$. Si cualquiera de las dos ramificaciones hubiera sido el único efluente, habría sido la corriente principal continua. El que una ramificación $B$ sea la continuación de la misma vida como una corriente principal $M$, depende, en consecuencia, de que otra ramificación $B^{\prime}$ esté presente también. Pero semejante ramificación adicional parecería algo extrínseco a la relación entre $B$ y $M$, y también algo relativamente trivial desde el punto de vista del interés propio, o permitásenos darlo por supuesto por mor del argumento. Así, volviendo al transplante de cerebro antes mencionado, demos por sentado que es de poca importancia para nuestro propio interés que nuestro otro hemisferio resulte o no ser canceroso después de todo, y sea transplantado, consecuentemente, a un cuerpo sano. Si esto es así, parece tener poco fundamento temer tal resultado.

Hay, por otra parte, una segunda vía por la que la sobrevivencia personal puede ser considerada dependiente de algo trivial, dada una explicación sobrevivencia como continuidad. Para la continuidad entre las etapas $V$ y $V^{\prime}$ de una vida se requiere que haya etapas intermedias $V_{1}, \ldots, V_{n}$, cada una fuertemente "conectada" a sus vecinas, donde la diferencia entre conexiones suficientemente fuertes y lo que simplemente se queda apenas corto parecería trivial, al menos en ocasiones.

Hemos visto la conclusión extraída a partir de tal razonamiento, según la cual la sobrevivencia ordinaria no debería importar verdadera y fundamentalmente. Y, además, hemos visto argumentar que lo que verdadera y fundamentalmente importa en nuestra sobrevivencia personal es solamente la "sobrevivencia", a saber, la continuidad con o sin ramificaciones equilibradas. (Esto tiene que ver con lo extrínseco y con la trivialidad inherente a si hay o no ramificaciones equilibradas adicionales. Pero no tiene que ver con la trivialidad inherente al corte 
constitutivo de la conectividad mínima. Obviamente tampoco queda eliminado este problema admitiendo cualquier fuerza de conectividad, sin importar cuán débil sea. Ya que aunque las diferencias de un penique son generalmente triviales, no es obvio que la diferencia entre cero peniques y un penique pueda evitar la trivialidad.)

Tal razonamiento es cuestionable por una ambigüedad decisiva. Cuando se dice que es "... de poca importancia para el interés propio si nuestro otro hemisferio resulta o no canceroso después de todo, y es transplantado, consecuentemente, a un cuerpo sano", ¿quién es el sujeto en cuestión y cuál es el tiempo que se ha fijado como meta? ¿Es acaso el protagonista de $M$ antes de la operación, antes de la coyuntura $J$, o es más bien el protagonista de $E$ después de la operación, en un momento posterior a la coyuntura $J$ ? Si es esto. último, entonces el sujeto en cuestión debería alegrarse por sí mismo de que las cosas sean como son, cualesquiera que sean, con respecto a la presencia o ausencia de ramificaciones equilibradas, ya que si hubiesen sido diferentes en ese respecto, entonces ese sujeto no habría existido. De esto no se sigue que el protagonista de $M$ anterior a la coyuntura $J$ deba igualmente alegrarse de que las cosas van a resultar como van a resultar, independientemente de cómo resulten de hecho. Ni tampoco se sigue siquiera que el protagonista de $E$ conservará, después de la coyuntura $J$, esta actitud: "Independientemente de si mi vida presente está equilibrada con otras ramificaciones, no habría tenido ninguna importancia para mí el que las cosas resultasen lo opuesto". Una vez admitido, esto último puede parecer sorprendente. El que alguien más con un origen cualquiera, pueda, en un momento dado, estar vivo, puede parecerle de poca o ninguna importancia esencial para alguien en ese momento. Sin embargo, compárese el hecho de que si los padres de uno nunca hubieran existido, entonces uno tampoco hubiera existido. Además, las consecuencias lógicas y analíticas pueden ser inesperadas aun para el más rápido y astuto de nosotms. 
Más seriamente cuestionable en el presente argumento es, de cualquier modo, la suposición de que lo importante no puede "depender" de lo "trivial" o "extrínseco", o sea, de lo que en sí mismo es "trivial" o "extrínseco". Esta pregunta fue formulada antes y se vuelve a enfatizar ahora apelando a ejemplos concretos como los siguientes.

La propiedad exclusiva de un objeto normalmente implica el derecho de uno a disponer de él de varias maneras sin que nadie más disfrute de un derecho similar. ¿Qué hay de la relación pertinente que uno mantiene con un objeto cuando uno es su propietario exclusivo? Considérese el hecho de que nadie más esta relacionado de forma similar con el objeto. ¿Es ese hecho "intrínseco" o "extrínseco" a la relación pertinente que uno mantiene con el objeto? Si decimos "intrínseco", entonces es un misterio por qué la inexistencia de una ramificación efluente rival no puede ser de este modo "intrínseca" a la relación pertinente de uno con el efluente a través del cual uno sobrevivirá. $\mathrm{Si}$, por el otro lado, el hecho de que nadie más posea el objeto (que uno posee en exclusiva) es considerado "extrínseco" a la relación pertinente de uno con dicho objeto, que a uno le interesa profundamente, entonces una relación importante puede, después de todo, depender de un rasgo "extrínseco" a ella y retener a la vez su propia importancia. Y uno se ve llevado a preguntarse por qué la auténtica sobrevivencia no puede ser otra de estas relaciones. (Un ejemplo similar lo ofrece la relación de autoría no anticipada, tan cara para la gente creativa en muchos terrenos de la vida; véase el libro de James Watson, The Double Helix para una explicación detallada. Pero el patrón básico es ubicuo: considérese el matrimonio y todo tipo de competencias y premios.)

Recordemos después el caso de la cubicidad, una forma que puede estar dotada de un significado y una atracción poderosos. (Compárese la fascinación griega por la circularidad.) Aun así, la dodecaedridad puede no tener en sí misma ninguna importancia para uno, y la diferencia entre doce y dieciséis aristas 
puede no importar en absoluto. Uno quiere un cubo, pero no le preocupa de ninguna manera si tiene doce o dieciséis aristas. Uno se inclina a estimar que los cubos tienen dieciséis aristas, pero nunca ha estudiado el asunto, y si resultan tener doce aristas, está bien. De cualquier modo, lo que uno quiere es un cubo. Y lo mismo vale para el número de caras. Uno se inclina a pensar en cubos de seis caras, pero lejos está de la certeza y no importaría si resultan ser cubos de ocho caras. Y algo semejante sucede con el número de ángulos. (Quizá no podríamos estar equivocados respecto de todas estas cosas al mismo tiempo sin perder la captación del concepto mismo de cubo. Yo mismo tiendo a dudarlo, y me inclino a pensar que una buena capacidad de reconocimiento sería suficiente, pero concedamos que así es por mor del argumento. Aun así, parece haber pocas dudas de que uno puede estar equivocado sobre alguno de los tres —aristas, ángulos o caras- estando a la vez en lo cierto acerca de los otros dos.)

Sin embargo, un cubo puede ser definido como un sólido regular con doce aristas rectas e iguales, o con seis caras cuadradas iguales. Tómese la opción elegida como análisis, y aun así se puede desear el analisandum sin desear el analisans. Pequeñas diferencias en el número de caras o aristas pueden ser cuestiones triviales que no nos conciernen aun cuando tener un cubo sea el deseo más anhelado. A la luz de este ejemplo, parece claro que algo importante puede depender de algo en sí mismo trivial. No se responda que lo "trivial" se ve revestido de importancia a través de la dependencia de lo importante respecto de él. Ya que esto sólo daría lugar a la respuesta de que la no-ramificación es, después de todo, importante ya que resulta crucial para nuestra sobrevivencia misma. Para que la apelación a la trivialidad de la no-ramificación no resulte una petición de principio, esa trivialidad debe reflejar la importancia "en sí misma" e independientemente de las conexiones ignoradas y desconocidas con lo que uno ya considera importante (quizá correcta y racionalmente). Pero entonces, de 
nuevo, el amante de los cubos puede anhelar un cubo sin importarle cuántas aristas tenga, lo que es, por lo tanto, "en sí mismo" un asunto trivial para él. Y tenemos el resultado de que lo importante puede depender después de todo de lo trivial.

Puede objetarse, finalmente, que dado que la sobrevivencia o la identidad personal consiste o superviene en, o ha de ser analizada con referencia a, el mantenimiento de ciertas relaciones, por lo tanto la sobrevivencia no debe importar o bien debe importar en una forma que requiere de una explicación por referencia a otras relaciones. ${ }^{16}$ Respuesta: "Importa" puede significar ya sea "importa realmente" o "debe importar" o cosas por el estilo. De cualquier manera, no resulta claro por qué o en qué sentido el analisandum debe importar siempre derivativamente a partir del analisans. Recuérdese el ejemplo de la cubicidad antes mencionado. Nuestra discusión de ese ejemplo y nuestra comparación de él con la sobrevivencia personal plantean una cuestión acerca de nuestra preocupación: ¿acaso ésta fluye o debe fluir a partir del analisans y hacia el analisandum y nunca en dirección opuesta? Necesitamos mostrar por qué, en el caso de la sobrevivencia personal, nuestra preocupación no puede propiamente fluir del analisandum hacia el analisans y extenderse a partir de la sobrevivencia hacia la no-ramificación. ${ }^{17}$

$16 C f$. Perry, 1976, principio de la p. 68: "Que yo voy a ser arrollado por un camión significa, dice Locke, que la persona que será arrollada por un camión recondará haber pensado y hecho lo que yo estoy pensando y haciendo ahora. Pero, ¿por qué habría yo de preocuparme especialmente por eso? ¿Por qué una persona que está teniendo semejantes recuendos ha de tener más importancia para m! que cualquier otra? Uno se ve inclinado a responder 'porque tener tales remembranzas es simplemente ser tú mismo'; pero ahora la explicación toma un camino equivocado..." Compárese también Parfit, 1984, Parte III, passim; e.g., p. 478: “Dado que la identidad personal a través del tiempo consiste únicamente en el mantenimiento de ciertas relaciones, lo que importa no es la identidad sino algunas de estas relaciones".

17 Agradezco a los múltiples auditorios que ofrecieron útiles discusiones de estas cuestiones, especialmente en las sesiones de la Universidad 


\section{REFERENCIAS}

Castañeda, Héctor-Neri, 1975, “Identity and Sameness", Philosophia, pp. 121-150.

Chisholm, R. M., 1976, “Identity Through Time”, cap. III de Person and Object, Allen \& Unwin, Londres. 1986, "Self-Profile", en Radu Bogdan (ed.), Roderick M. Chisholm, D. Reidel, Dordrecht.

Johnston, Mark, 1987, "Human Beings", Journal of Philosophy, pp. 59-84.

Lewis, David, 1976, “Survival and Identity”, en Amelie Rorty (ed.), The Identities of Persons, University of California Press, Berkeley, pp. 17-40.

, 1983, "Proscripts to 'Survival and Identity'", Philosophical Papers, Oxford University Press, Oxford, pp. 73-77.

Nozick, Robert, 1981, Philosophical Explanations, Harvard University Press, Cambridge, Mass.

Parfit, Derek, 1976, "Lewis, Perry and What Matters", en Amelie Rorty (ed), The Identities of Persons, University of California Press, Berkeley, pp. 91-107. [Versión española: Lewis, Penry y lo que importa, trad. de Álvaro Rodríguez Tirado, Cuadernos de Crítica, núm. 39, Instituto de Investigaciones Filosóficas, México, 1985.]

1984, Reasons and Persons, Oxford University Press, Oxford.

Perry, John (ed.), 1975, Personal Identity, University of California Press, Berkeley.

- 1976, "The Importance of Being Identical", en Amelie Rorty (ed.), The Identities of Persons, University of California Press, Berkeley, pp. 67-91.

Quine, W. V., 1953, "Identity, Ostension, and Hypostasis", From

de Nueva York, la Sociedad Argentina de Análisis Filosófico, la Universidad Trinity, la Universidad de Helsinki, la Universidad Mass-Amherst, la Universidad de Miami, la Universidad de Witwatersrand, y mi seminario en la Universidad de Brown. Un especial agradecimiento a Simon Blackburn, Héctor-Neri Castañeda, Terence Horgan y Derek Parfit, por sus amplios comentarios escritos. Estoy también agradecido por la útil discusión, oral o escrita, con Mark Johnston, Jaegwon Kim, David Pears, Theodore Scaltsas, Sydney Shoemaker, David Sosa, Timothy Sprigge y Peter Unger. 
a Logical Point of View, Harvard University Press, Cambridge, Mass.

Shoemaker, Sydney and Richard Swinburne, 1984, Personal Identity, Basil Blackwell, Oxford.

Sosa, Ernest, 1983, "Classical Analysis", Journal of Philosophy, pp. 695-710.

L, 1987, "Persons and Other Beings: Subjects Among Other Things", Philosophical Perspectives I: Metaphysics, pp. 155-189. Wiggins, David, 1980, Sameness and Substance, Harvard University Press, Cambridge, Mass.

Williams, Bernard, 1973, Problems of the Self, Cambridge University Press, Cambridge. [Versión española: Problemas del yo, trad. de J. G. M. Holguera, Instituto de Investigaciones Filos6ficas, UNAM, México, 1986.]

Racibido: 2 febraro 1990.

Traducción de Gustavo ORTIz MuLáN 


\section{SUMMARY}

Section I introduces to "the paradox" in the following way: "Life may turn sour and, in extremis, not worth living. On occasion it may be best, moreover, to lay down one's life for a greater cause. None of this is any news, debatable though it may remain, in general or case by case. Now comes the news that life does not matter in the way we had thought. No resurgence of existentialism, nor tidings from some ancient religion or some new cult, the news derives from the most sober and probing philosophical argument (the extraordinary Parfit in Part III of his Reasons and Persons), and takes more precisely the following form:

"The Paradox: Even though life $\mathrm{L}$ is optimal (in all dimensions), and even though if it were extended $L$ would continue to be optimal, it does not follow that it is best to extend it, even for the subject whose life $L$ is."

Section II defends a certain view of the nature of persons and personal identity. Assuming that (1) one is not a soul, one's existence does not consist in the existence of any soul, and one's predurance does not consist in the perdurance of any soul, and that (2) one is not any body or collection or particles, one's existence does not consist in the existence of any body or collection of particles, and one's perdurance does not consist in the perdurance of any body or collection of particles, an aristotelian answer to the question "what then is the person, and what might constitute its existence and perdurance?" is offered, divided into three parts:

1. The personal profile of a person on an occasion is a combination of a psychological profile (experiences, thoughts, actions, beliefs, desires, virtues, abilities, et cetera) and a physical profile including whatever properties of appearance or physical prowess may be thought significant for the identity of a person.

2. The existence of a person on an occasion consists in the embodiment by a body of a personal profile, on that occasion.

3. The life of a person through a sequence of occasions is a sequence of embodied personal profiles, occasion by occasion, interrelated by causal links such as realized intentions, true memories, and the physical mechanisms of growth and aging. The perdurance of a person across a sequence of occasions consists in the embodiment of a personal profile by a body on each of those occasions, and in the appropiate causal linkage amongst those embodiments. 
Four different objections to this thesis of survival as continuity are commented upon and evaluated.

Section III argues for the Paradox on that basis, and reflect on its philosophical implications and on the options it presents. In a first step, the discussion delineates three distinct concepts of $m y$ surviving and studies the relations between them: (1) my surviving as "my life's extending without branching, by means of appropiate causal relations linking present and past stages to future stages"; (2) my 'surviving', explained thus: "that my influence extend by means of appropiate causal relations linking present and past stages of my life to future psychological states, with or without branching", and (3) my "quasi-surviving": "that the sequence of psychological states constitutive of my past and present life be followed by further psychological states with a certain 'similarity', whether accompanied or not by either causal influence or nonbranching".

In the second part, two lines of argument both leading to the conclusion that survival is a misvalued commodity are presented and discussed. They are as follows:

First line:

Premise 1: The worth of personal survival must be "derivative", and must derive from the concern that we invest in our "nonprivate" projects.

Premise 2: But one's "nonprivate" projects would sometimes be advanced even better by a team of duplicate "survivors" than by a solitary one.

Conclusion: Sometimes it would be better for one's life to branch (yielding two or more duplicate "survivors") than for it not to branch (yielding only a solitary survivor).

Second line:

Premise 1: What matters in the personal survival of a continuing life (a) cannot depend on features "extrinsic" to the pertinent relation among its stages, and (b) cannot depend on any "trivial" fact.

Premise 2: (Suppose the main stream $M$ of a life arrives at a certain juncture J. And consider how that main stream may be related to an effluent $E$ flowing from main stream $M$ at juncture $J$. Are there other effluents, "equipoised" to $E$, which also flow from $M$ at J?) Whether or not there are such effluents is a matter that must be "extrinsic" to the relation between $M$ and $E$ in virtue of which $E$ might constitute the continuation of $M$; moreover, it is a matter that must be "trivial" to the protagonist of that mainstream $M$. 
Conclusion: What matters in the survival of life $M$ through an effluent $E$ beyond that juncture J (or, equivalently, what matters in the survival of the protagonist of $M$ through $E$ ) cannot involve or depend upon whether or not there are other effluents flowing out of $M$ at $J$, and equipoised to $E$.

$[R$. 